\title{
Correction to: Design and Application Research of Targeted Persons Control System Based on Big Data
}

Jinbo Wu, San-you Zhang, and Zhiguo Yan

\begin{abstract}
Correction to:
Chapter "Design and Application Research of Targeted Persons Control System Based on Big Data" in: V. Sugumaran et al. (Eds.): Application of Intelligent Systems in Multi-modal Information Analytics, AISC 1234, https://doi.org/10.1007/978-3-030-51556-0_67
\end{abstract}

In the original version of the book, the following belated correction has been incorporated: The co-author's name Zhiguo Yan has been included and the acknowledgement section has been modified in Chapter 67. The chapter and book have been updated with the change. 